\title{
Spectrum Aggregation: Overview and Challenges
}

\author{
Wei Wang ${ }^{\ddagger}$ (corresponding author), Zhaoyang Zhang ${ }^{\dagger}$, Aiping Huang ${ }^{\dagger}$ \\ † Department of Information Science and Electronic Engineering, \\ Key Laboratory of Integrate Information Network Technology, \\ Zhejiang University, Hangzhou 310027, P.R. China \\ ‡ Key Laboratory of Information Coding and Transmission, \\ Southwest Jiaotong University, Chengdu 610031, P.R. China \\ E-mail: wangw@zju.edu.cn
}

\begin{abstract}
Spectrum aggregation has recently received much attention due to the quick increasing of services. This technique makes it possible for multiple spectrum bands to be utilized by the same user in order to satisfy the large bandwidth demand of the service and achieve better performance. This paper provides an overview on spectrum aggregation. The research progresses on spectrum aggregation for LTE-Advanced and dynamic spectrum access are briefly described respectively. Finally, some research challenges for protocols and algorithms are addressed for future studies.
\end{abstract}

Keywords: spectrum aggregation, LTE-Advanced, dynamic spectrum access

Corresponding to: Wei Wang, Institute of Information and Communication Engineering, Zhejiang University, Zheda Rd. 38, Hangzhou 310027, P.R. China.

This work is supported by National 973 Program under grant No. 2009CB320405, National Natural Science Foundation of China under grant No. 60972057, 60972058 and 60802012, Chinese Universities Scientific Fund under grant No. 2009QNA5029, Scientific Research Fund of Zhejiang Provincial Education Department under grant No. Y200909796, and the open research fund of Key Laboratory of Information Coding and Transmission, Southwest Jiaotong University. 


\section{Introduction}

With the rapid development of communication technologies, the demand of radio spectrum is increasing quickly as a rare and valuable resource. It is difficult to satisfy high bandwidth requirements of various kinds of bandwidth services supported by wireless communication systems. Traditional wireless communication techniques can only support the utilization of continuous spectrum resource, but the wide continuous spectrum bands are rarely available under the current situation of spectrum resource and the policy of stable licensed spectrum utilization.

The motivation of spectrum aggregation is to utilize discrete spectrum bands to support high bandwidth requirements of services. In this way, the discrete spectrum bands can sustain information flow transmission service as the same as the continuous spectrum bands. Cognitive radio [1,2] can sense and use idle spectrum bands by inspecting radio environment intelligently. With this sensing ability, the combination of narrow idle spectrum bands becomes possible using spectrum aggregation.

Spectrum aggregation technology is studied by many companies and standard organizations all over the world. QinetiQ [3] is one of the research organizations trying to take advantage of spectrum aggregation technique to sustain broadband services, and study the causes of spectrum fragmentation, the utilization principles and the realization methods. As one of the key technologies of LTE-Advanced [4], spectrum aggregation is attracting increasing attention from Ericsson, NTT Docomo, ETRI, China Mobile, and so on. Meanwhile, Federal Communications Commission (FCC) is also preparing to eliminate the limit of spectrum utilization for flexible utilization of spectrum resource [5], which releases the preventers of spectrum aggregation. The spectrum aggregation technology is also standardized for Long Term Evolution Advanced (LTE-Advanced) based on the 3rd generation telecommunication (3G) systems.

A lot of researchers also have large interest in spectrum aggregation. A few publications appear in recent years. In 2005, Robinson improves the system capability and spectrum utilization efficiency greatly by taking advantage of spectrum trading, a scheme which allows the spectrum owners to exchange some parts of their own spectrum bands and aggregate the spectrum bands together [6]. In 2008, Zhang's research group proposes a discontiguous spectrum access and aggregation scheme-Discontiguous Orthogonal Frequency Division Multiplexing (DOFDM). With the DOFDM technology, discrete spectrum bands are aggregated, which can provides large bandwidth to the users with high requirement. They also propose an algorithm called Aggregation-Aware Spectrum Assignment (AASA) with spectrum aggregation ability taken into consideration [7]. The network capacity will be greatly improved if the DOFDM technology and resource allocation algorithms based on spectrum aggregation are applied to cognitive radio networks. Furthermore, they even consider the open spectrum aggregation and utilization with hardware limitations of sensing and transmission abilities in spectrum-sharing networks [8].

Considering the dynamics of spectrum availability, [18] provides the prediction-based spectrum aggregation scheme for decreasing the overhead of re-allocation. One is Maximum 
Satisfaction Algorithm (MSA) for admission control. The main idea is assigning spectrum for the user with larger bandwidth requirement first and leaving better spectrum bands for remaining SUs considering different bandwidth requirements of secondary users (SU). The other is Least Channel Switch (LCS) strategy for spectrum assignment considering the dynamic access of SUs with different bandwidth requirements. In order to decrease the times of channel switch at sensing moments, the concept of outage probability is introduced to predict the probability that the spectrum band will not be able to provide the current capacity.

The technology of spectrum aggregation is of important research and application value. Currently, the research works in this area have just started. The theoretical and technical problems are waiting to be solved. Almost all work done in research works assume that all spectrum bands in spectrum aggregation have the same property while different spectrum bands differ greatly in real networks, so the problem is how to provide homogeneous information communication by taking advantage of discontiguous spectrum bands with different properties. Considering the utilization of discontiguous spectrum bands with different properties to fulfill bandwidth requirement of users, the most difficult tasks of the problem are maximizing information flow as well as ensuring reliability of information flow by spectrum aggregation and scheduling. Based on these aims, an ideal scheduling scheme is necessary to facilitate the communication using discontiguous spectrum bands with the same average error and delay performance as that using contiguous spectrum communication in a statistical sense. Spectrum aggregation is a communication method which makes transmission of integral information flow through discontiguous spectrum bands with different properties possible.

The rest of the paper is organized as follows. Section 2 introduces some spectrum aggregation technologies briefly. Section 3 provides the progress of spectrum aggregation in LTE-Advanced systems, while Section 4 provides the algorithms of spectrum aggregation for dynamic spectrum access scenarios. In Section 5, the challenges are discussed from both the protocol and algorithm perspective. Section 6 concludes this paper.

\section{Key Technologies}

One of the key technologies of spectrum aggregation is the physical layer aggregation scheme, e.g. DOFDM. Wireless transceivers are becoming more and more versatile, powerful, and portable with the rapid evolution of microelectronics. This has enabled the development of software-defined radio (SDR) technology which is now crucial in cognitive radio technology. A flexible modulation technique based on multicarrier modulation called non-contiguous multicarrier modulation (NC-MCM) [9] can turn off the subcarriers which would interfere with incumbent transmissions. DOFDM can be realized by modifying the efficient block processing of Orthogonal Frequency Division Multiplexing (OFDM) [10,11]. The proposed modification would have multiplexing of OFDM subcarriers align with the spacing of the channels which are being used. It would be possible to transmit on vacant channels and avoid active ones by placing data and zeros in the appropriate IFFT bins [12]. Therefore, the spectrum aggregation ability is directly related to the possibility of 
modification.

The other important technology of spectrum aggregation is scheduling, which tries to achieve the same performance as contiguous spectrum bands. The communication equipments have to assign spectrum resource to transmit information flows in transmitter terminal and combine information flows from different spectrum bands reliably in receiver terminal. Larger spectrum band diversity caused by frequency range span is greatly challenging in the research of spectrum aggregation and scheduling mechanism. AASA is an efficient way to aggregation the discrete spectrum bands. With the assumptions that all users have the same bandwidth requirement, the proposed optimal scheme tries to utilize the first aggregation range satisfying the requirement from the low frequency side. The flow chart of AASA is described as Fig. 1.

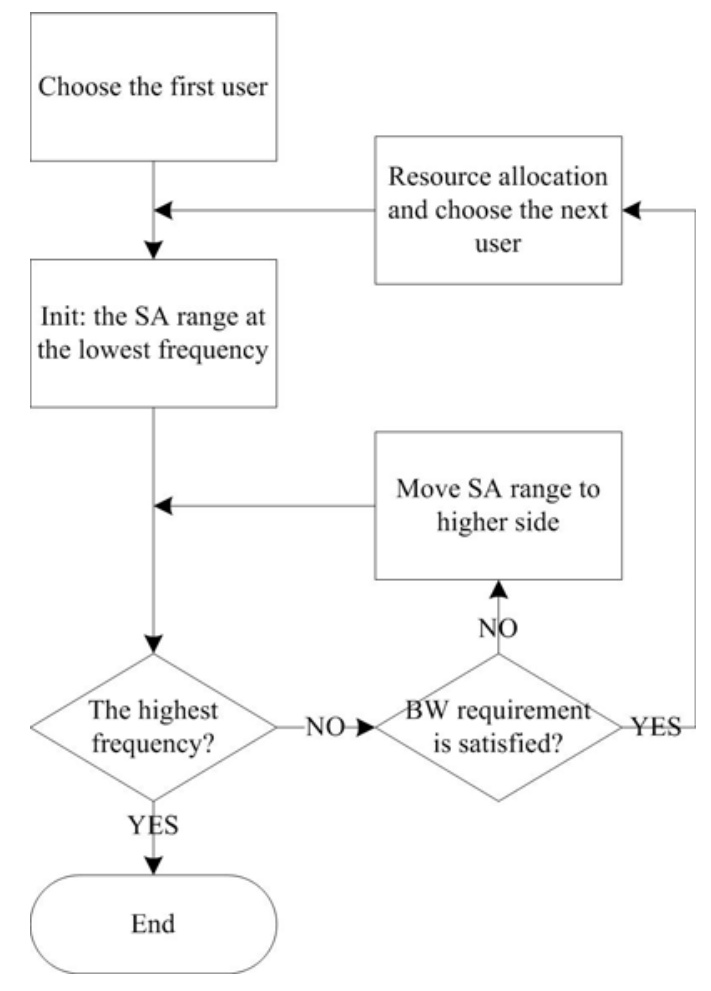

Fig. 1 Flow chart of AASA

Although the above two solutions can address these key technologies in general cases, they may not works well for some scenarios, especially for LTE-Advanced and dynamic spectrum access cases. For LTE-Advanced systems, more practical issues need to be considered, while for dynamic spectrum access systems, the dynamics of spectrum availability is an important issue. Table I provides the comparison at several aspects of the existed schemes on spectrum aggregation. 
Table I The existed schemes on spectrum aggregation

\begin{tabular}{llllll}
\hline & DOFDM & AASA & LTE CA & MSA & LCS \\
\hline Aggregation Layer & PHY & MAC & PHY/MAC & MAC & MAC \\
Scenarios & General & General & LTE-Advanced & DSA & DSA \\
Large-scale Spectrum & No & Yes & No & Yes & Yes \\
Spectrum Dynamics & No & No & No & No & Yes \\
Multiple Users & $\begin{array}{l}\text { Single/multiple } \\
\text { users }\end{array}$ & $\begin{array}{l}\text { Multiple user with } \\
\text { the same bandwidth } \\
\text { requirement }\end{array}$ & $\begin{array}{l}\text { Single/multiple } \\
\text { users }\end{array}$ & $\begin{array}{l}\text { Multiple } \\
\text { users }\end{array}$ & Single/multiple \\
& & Low & Low & Normal & High \\
\hline Complexity & Normal & Low & & & \\
\hline
\end{tabular}

\section{Spectrum Aggregation for LTE-Advanced}

With the development of mobile communication technology, both communication traffic volume and data throughput keep growing, which results in that $3 G$ system can not satisfy some users' bandwidth requirements any more. In order to improve the capacity of wireless communication and get well prepared for communication business which is getting more and more diversified, LTE has been proposed instead of 3G system, which will easily obtain reduced latency, higher user data rates, cost-reduction, improved system capacity and coverage. As further evolution of LTE, LTE-advanced system demands more on lots of key technology parameters [13]. Therefore, more candidate technologies will be brought in, such as spectrum aggregation, relay, MIMO and so on. Besides, LTE-advanced system supports compatibility with LTE system, both forward and backward.

During the evolution from LTE to LTE-advanced, the most influential factor is the demand for wider radio spectrum band. As a result, 3GPP proposed spectrum aggregation technology (which is also called carrier aggregation in LTE-advance standardization) [14]. It aggregates several carriers into one wider channel, even though these spectrum fragments are discontiguous. What is more important is that spectrum aggregation technology makes a great contribution to the compatibility between LTE and LTE-advanced system.

The bandwidth that LTE-advanced system is able to support is $100 \mathrm{MHz}$, while LTE system can only support 20MHz. Nowadays, the lack of radio spectrum has become the bottleneck of the development of wireless networks, and as a result it's obviously difficult to find a spectrum band in LTE system which is wide enough for LTE-advanced system. Consequently, it is urgent for us to figure out a way to gain wider radio spectrum band. The answer is spectrum aggregation technology. [15] 
On the method of spectrum aggregation, several carriers in LTE system can be aggregated into one wider channel in LTE-advanced system which is wide enough for $100 \mathrm{MHz}$, even though these carriers in LTE are in different frequency bands. On the aggregated wider channel, LTE-advanced terminal can access several spectrum fragments simultaneously, and meanwhile LTE terminal can access only one spectrum fragment of them, which not only meets the needs of spectral compatibility, but also reduces the cost of bits. On the other hand, in consideration of actual deployments of LTE-advanced system, spectrum aggregation technology has an obvious advantage. Because of the direct aggregation of LTE carriers, there's no need to make large changes in the physical layer of LTE system, which reduces the design difficulty of LTE-advanced system greatly.

The research progress of spectrum aggregation mainly focuses on the following aspects.

1) Design of guard bandwidth between carriers [16]

When the aggregated LTE carriers are in contiguous frequency bands, it is a wise decision to reduce the guard bandwidth between carriers reasonably, which can obviously improve the spectrum utilization efficiency.

2) Selection of carrier bandwidths [17]

In the process of spectrum aggregation, it should be taken into consideration that both the aggregated carriers in LTE and the wider channel in LTE-advanced have different bandwidth. Consequently, there are several methods of aggregation, e.g. $30 \mathrm{MHz}=20 \mathrm{MHz}+10 \mathrm{MHz}=$ $20 \mathrm{MHz}+5 \mathrm{MHz}+5 \mathrm{MHz}$. In order to reduce the design complexity of transceivers, a concept is proposed called bandwidth factor, which indicates the multiple relationship between bandwidth of aggregated carriers in LTE, e.g., when the bandwidth factor is 2, we can only choose the solution that $30 \mathrm{MHz}=20 \mathrm{MHz}+10 \mathrm{MHz}$, for 20 is twice as large as 10 . Obviously, the proposal of bandwidth factor can restrict the methods of aggregation successfully and reduce the design complexity of transceivers greatly.

\section{3) Aggregation of data stream [16]}

In the process of spectrum aggregation, each carrier corresponds to independent data stream. In the sight of lower layers, there are two alternative schemes of data stream aggregation as shown in Fig. 2.

After comparing these two methods, it can be found that the MAC layer aggregation scheme is easier to fulfill the smooth transition from LTE system to LTE-advanced system, which is the most important key factor that should be considered. In view of the follow-up development of spectrum aggregation technology, the data stream should be aggregated in MAC layer. 


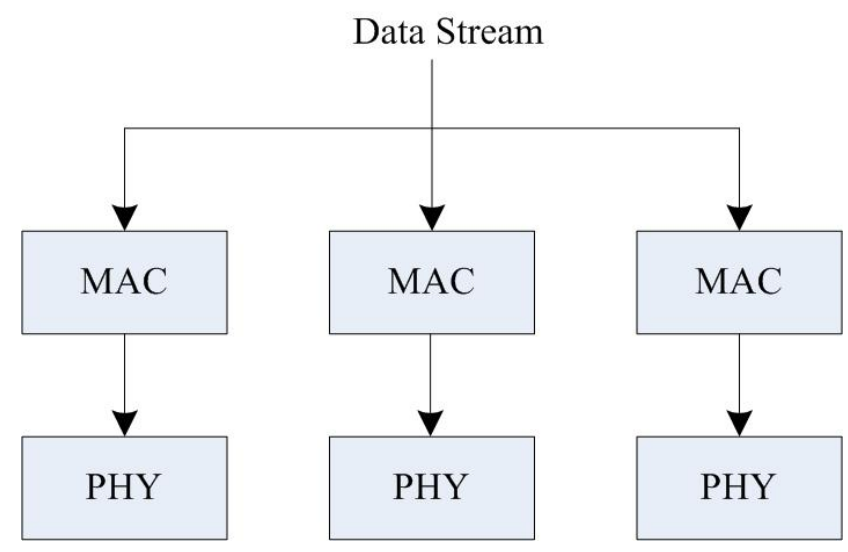

(A) Data streams are aggregated in MAC layer

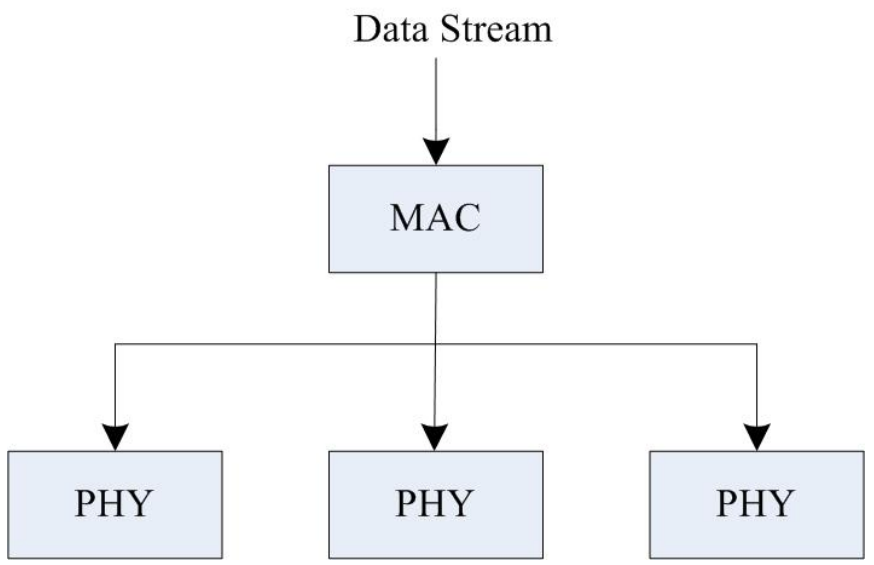

(B) Data streams are aggregated in physical layer

Fig. 2 Two aggregation methods of data streams

4) Design of control channels [17]

As an important part of LTE-advanced system, the design of control channels should be considered fully and carefully in the technology of spectrum aggregation. There are also two alternatives as shown in Fig. 3, in which each parallelogram represents one carrier, and the filled areas represent control channels.

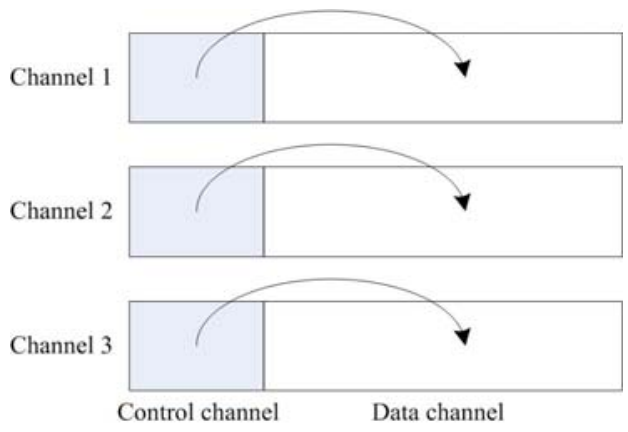

(A)

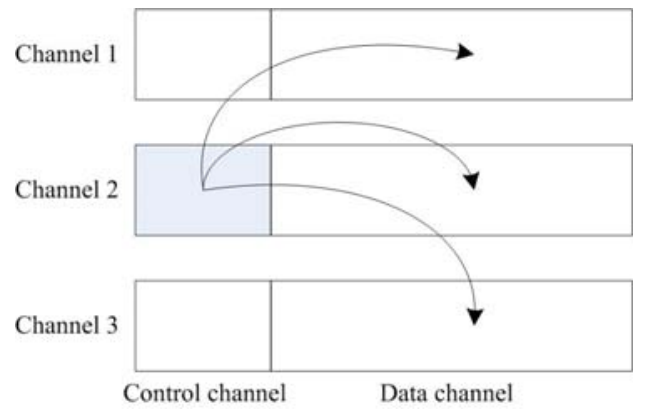

(B)

Fig. 3 Two kinds of control channels 
Comparing the two control schemes, the scheme A has several advantages. Firstly, the scheme A can save a lot of power dissipation and cost of bits, because the control information of the spectrum band is just in this band. What's more, it can make the most of the existing system structure, which means a lot to the backward compatibility of the system.

\section{Spectrum Aggregation for Dynamic Spectrum Access}

For dynamic spectrum access based on cognitive radio, the spectrum's availability is time-varying. In this case, it have to be considered that the switch overhead of spectrum aggregation strategies.

On the spectrum aggregation scheme for dynamic spectrum access cases, we propose an admission control algorithm and a spectrum assignment strategy considering both the spectrum aggregation and the channel switch based on the state statistics of each channel [18]. Because of the limited hardware aggregation capability, only the fragments within a predefined region can be aggregated to be utilized by a user.

The aim of spectrum access of SUs is to satisfy as many SU bandwidth requirements as possible to improve the spectrum efficiency. The main idea of the MSA algorithm is utilizing the worst spectrum band which can just satisfy the bandwidth requirement of this SU in consideration of the upcoming users.

Based on the main idea of MSA, the following rules are given.

1) The narrower regions are "worse" than wider ones since they are less capable of accommodating other upcoming SUs.

2) The SUs with higher bandwidth requirement is more difficult to be assigned an appropriate spectrum bands. So the SUs should access to the spectrum band in descending order of their bandwidth requirements.

3) If possible, the SU should access to the left edge of the spectrum region.

The detail of the MSA algorithm is provided as a flow chart in Fig. 4.

For LCS algorithm, the outage probability $\delta$ is defined firstly as the probability that the spectrum bands within the aggregation region can not fulfill the bandwidth requirement. The outage probability denotes the probability that the channel-unit in the aggregation region switches to a state with lower capacity, while current bandwidth is $R$. i.e. after $H$ transitions, the probability that the state ends on the left side of $R$ state is just the outage probability.

Based on the periodic sensing, the SUs are aware of the dynamic alteration of spectrum status every time $T$. If the provided bandwidth can not satisfy the SU, then the channel switch should be implemented which causes overhead in the network. A strategy is needed to adjust the spectrum band assignment for minimizing the channel switch times. 


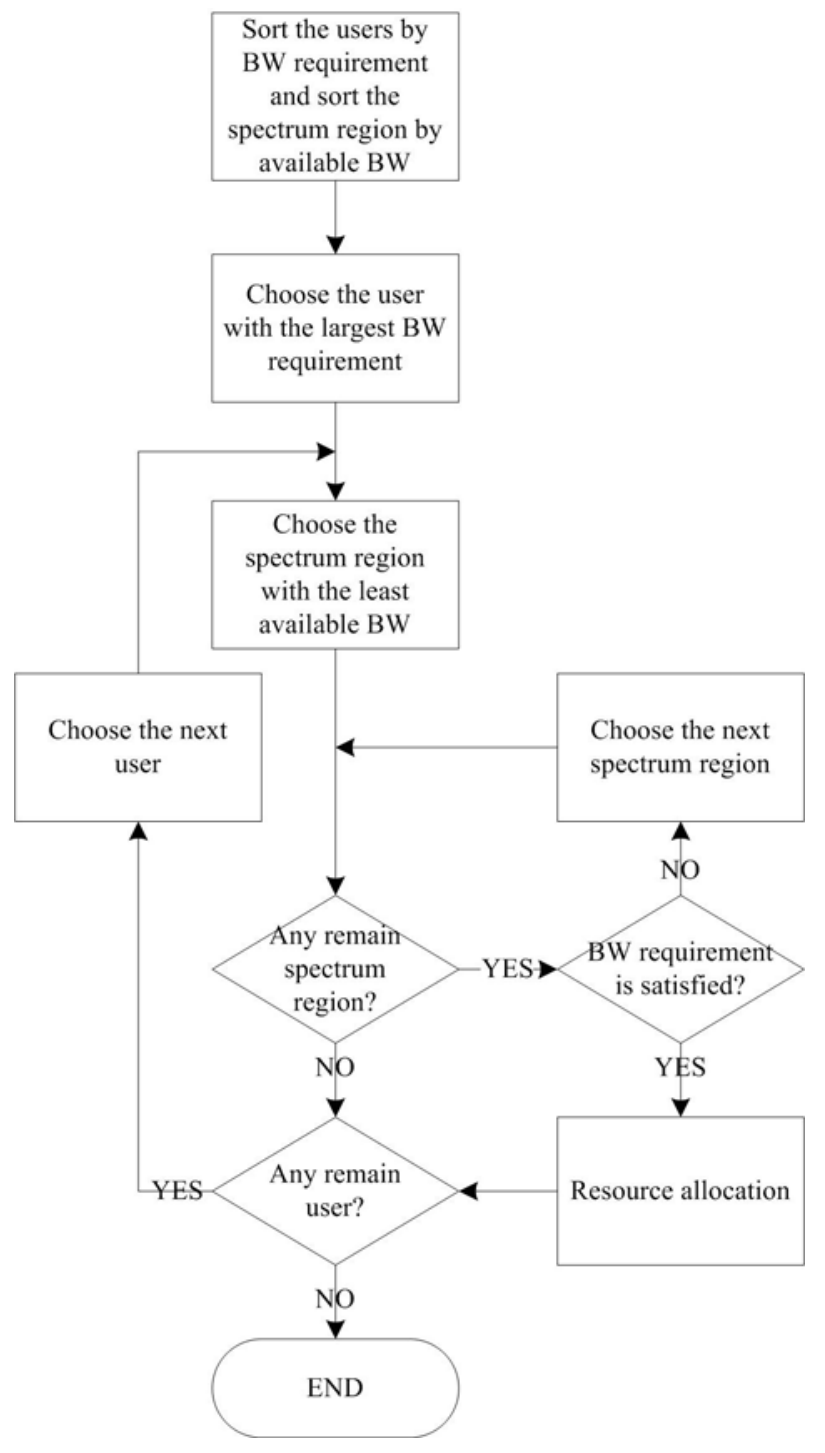

Fig. 4 Flow chart of MSA

The performance of MSA and LCS strategies is provided in Fig. 5 and Fig. 6, which can show the performance advantages of the MSA and LCS strategies. More detailed simulation results can be founded in [18].

From Fig. 5, when the network load is small, which means that the sum of all SUs' bandwidth requirements is low compared with the total spectrum bandwidth, the performance of the contiguous assignment scheme is almost the same as AASA and MSA. That is because the spectrum bandwidth is so large that the SUs' requirements can be satisfied just by simple mechanisms. But as the network load increases, both the AASA and MSA algorithms achieve much better performance than the contiguous assignment scheme for supporting more bandwidth requirements. Also, when comparing MSA and AASA, we could see that MSA achieves better performance when the provided spectrum bandwidth is not enough.

From Fig. 6, the channel switch times using LCS strategy are much fewer than the performance without LCS. The smaller the difference among SU requirements is, LCS 


\section{Macrothink}

achieves more improvement. Especially, when the difference is too large, it is difficult to find an appropriate part of spectrum with low outage probability for the SUs which need so many channel-units, even though the prediction-based method is adopted.

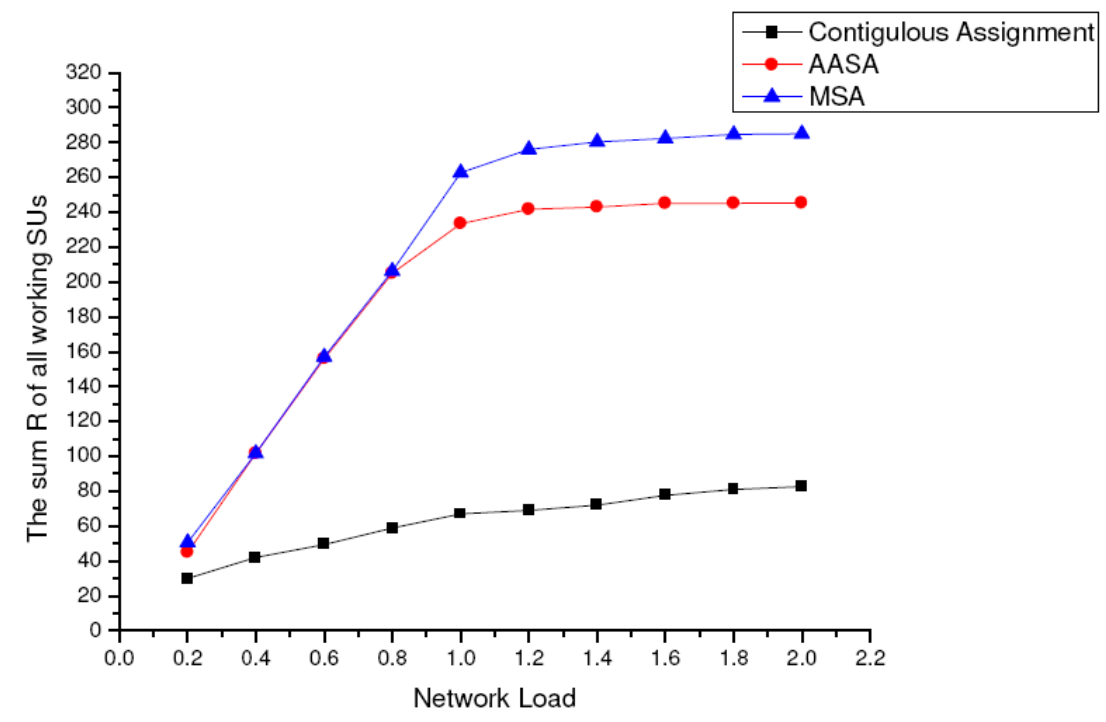

Fig. 5 The sum of the bandwidth of SUs accessed to the network [18]

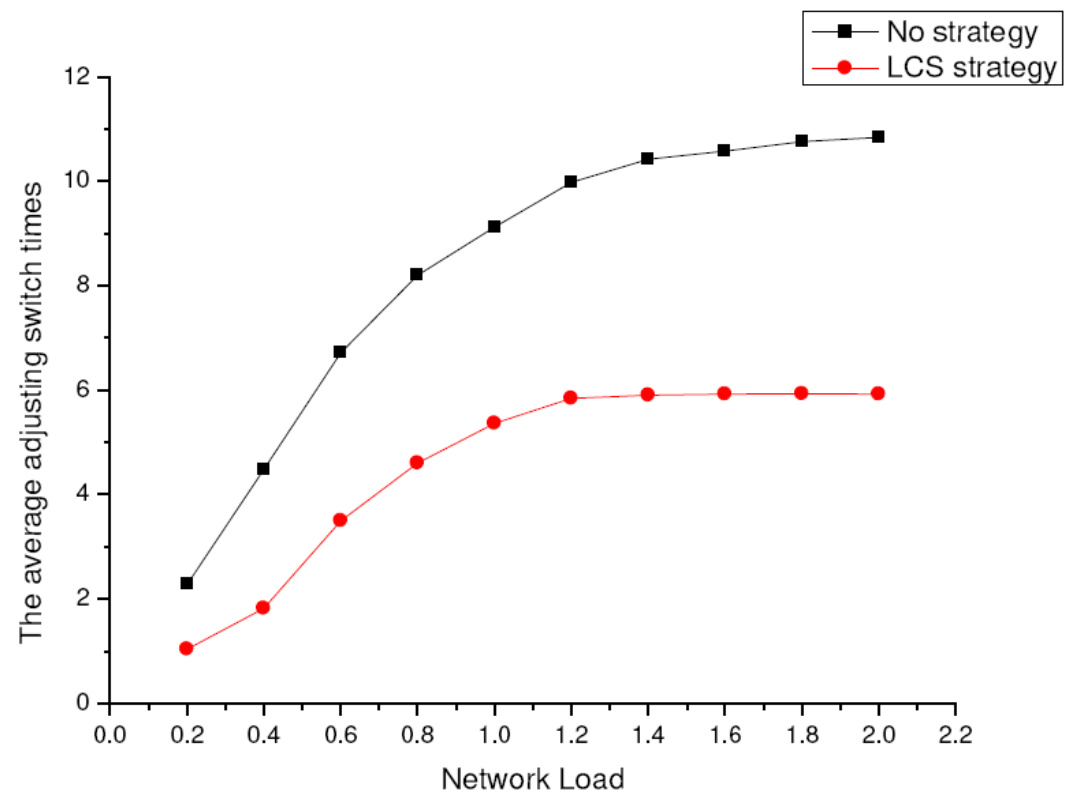

Fig. 6 Average channel-switch times according to the network load [18]

\section{Research Challenges}

This section provides several research challenges on both protocols and algorithms, as guidelines for future researches. 


\subsection{Challenges on Protocols}

\section{Challenge 1: How to design the control channels?}

For spectrum aggregation, additional control channels are needed. The status of multiple channels and the spectrum aggregation decisions need to be exchanged between nodes. It is necessary to design efficient control channels to transmit the larger mount of control information than the single spectrum band cases. If the overhead of control channels is too large, it is possible to transmit the information of only a part of spectrum bands, which can achieve the sub-optimal performance. This kind of control channel design balances the tradeoff between the performance and the system complexity.

\section{Challenge 2: How to exchange the spectrum information during handover?}

When handover, the target cell tries to find out whether the available resource is enough. For the spectrum aggregation case, the available spectrum resource can be increased by spectrum re-aggregation. In other words, the current available resource is not appropriate to be used as a factor for handover decision. A new handover protocol should be proposed to consider the potential access capability of target cells. The gain brought by resource re-allocation needs to be considered during the handover process.

Challenge 3: What are the extra overheads of cross-layer protocols for spectrum aggregation?

The implement of spectrum aggregation needs the cooperation between multiple layers. The lower layers (mainly Physical layer and Link layer) aggregate the spectrum bands and provide the equivalent bearer services to higher layers as contiguous spectrum bands. Additional information exchange about spectrum aggregation is needed between lower layers and higher layers.

\subsection{Challenges on Algorithms}

\section{Challenge 4: What is the balance of diversity-multiplexing tradeoff?}

The aggregation of multiple spectrum bands can be considered as multiple parallel channels. Similar to MIMO transmission, transmitting different data at the spectrum bands can increase the throughput, while transmitting the same date can improve QoS. The data transmission using spectrum aggregation also has the diversity-multiplexing tradeoff. From information theoretic perspective, the bound of the diversity-multiplexing tradeoff can be investigated.

\section{Challenge 5: How to schedule the users to transmit?}

For a given set of users and spectrum bands, the scheduling problem considering spectrum aggregation can be modelled mathematically as a combination optimization problem, which is a NP-hard problem. A sub-optimal scheduling scheme is needed with acceptable computational complexity.

Challenge 6: Can the equipments aggregate large-scale available spectrum? 
Because of the limited hardware aggregation capability, only the fragments within a predefined region can be aggregated to be utilized by a user. The central carrier frequency of the aggregation range becomes a new key parameter which can be configured during scheduling. Dynamic programming is a eligible way to determine the primary carrier frequency, but with too large computational complexity.

\section{Challenge 7: Can the spectrum aggregation save energy?}

If the transmission capability of available spectrum bands is more than it required, the users may aggregate more bands than it wants. In this way, the data can be dispersed into more bandwidth and the data rate of a bandwidth unit decreases, so that the energy efficiency is improved.

\section{Challenge 8: How to consider the user at the edge of cells?}

Because the signals of different frequencies have different propagation properties, some spectrum bands may not reach the edge of cells, which bring new challenges when scheduling. The spectrum bands with lower frequency should be allocated to the users at the cell edges with high priority. The fairness is also a new issue for the users at the edge of cells.

\section{Conclusions}

This paper provided the overview and challenges of spectrum aggregation. We conclude the research progress on spectrum aggregation for LTE-advanced system and dynamic spectrum access cases respectively. The technique challenges are discussed on both protocols and algorithms. These discussions will hopefully serve as guidelines of further research studies on spectrum aggregation.

\section{Acknowledgements}

The authors express their gratitude to Lingcen Wu and Furong Huang, who provide related materials for improvement of this paper.

\section{References}

[1] J. Mitola, G. Maguire, “Cognitive radio: Making software radios more personal”, IEEE Personal Communications, vol. 6, no. 4, pp. 13-18, Aug. 1999

[2] S. Haykin, "Cognitive radio: Brain-empowered wireless communications", IEEE Journal on Selected Areas in Communications, vol. 23, no.2, pp. 201-220, Feb. 2005

[3] QinetiQ, Ltd., “A Study of the Provision of Aggregation of Frequency to Provide Wider Bandwidth Services”, final report for Office of Communications(Ofcom), UK, QINETIQ/06/01773, Aug. 2006

[4] Hideshi Murai, et al., "LTE Advanced - The Solution for IMT-Advanced", IMT-Advanced Workshop, TTA, Jun. 2008 


\section{Macrothink}

Network Protocols and Algorithms

ISSN 1943-3581

2010, Vol. 2, No. 1

[5] Public Interest Spectrum Coalition, "Reply Comments of the Public Interest Spectrum Coalition”, FCC document, Dec. 2008

[6] D.L.Robinson, A.K. Shukla, J. Burns, A. Atefi, "Resource trading for spectrum aggregation and management”, IEEE SPAN 2005, pp. 666 - 671, Nov. 2005

[7] D. Chen, Q. Zhang, W. Jia, “Aggregation Aware Spectrum Assignment in Cognitive Ad-hoc Networks”, IEEE CrownCom 2008, pp. 1 - 6, May 2008

[8] J. Jia, Q. Zhang, X. Shen, "HC-MAC: A Hardware-Constrained Cognitive MAC for Efficient Spectrum Management”, IEEE Journal of Selected Areas in Communications, vol. 26, no. 1, pp. 106 - 117, Jan. 2008

[9] A.M. Wyglinski. "Effects of Bit Allocation on Non-contiguous Multicarrier-based Cognitive Radio Transceivers”. IEEE VTC 2006

[10]T. A. Weiss and F. K. Jondral, "Spectrum pooling: An innovative strategy for the enhancement of spectrum efficiency” , IEEE Commun. Mag., vol. 42, no. 3, Mar. 2004, radio Communications Supplement, pp. S8-S14.

[11]J. D. Poston and W. D. Horne, "Discontiguous OFDM considerations for dynamic spectrum access in idle TV channels”, in Proc. IEEE DySPAN 2005, pp. 607-610, Nov.2005, [12]D. J. Schaefer, “Wide area adaptive spectrum applications”, in Proc. IEEE MILCOM, vol. 1, Oct. 2001, pp. 1-5.

[13]3GPP TR 36.913 v8.0.0. "Requirements for Further Advancements for E-UTRA”, 2008

[14]3GPP REV-080030, Ericsson, “Technology components”, Apr 2008

[15]3GPP R1-082569, CATT, “Consideration on technologies for LTE-Advanced”, June 2008

[16]3GPP Rl-082448, Huawei, “Carrier aggregation in Advanced E-UTRA”, June 2008

[17]3GPP R1-082468, Ericsson, “Carrier aggregation in LTE-Advanced”, June 2008

[18]F. Huang, W. Wang, H. Luo, G. Yu, Z. Zhang, "Prediction-based Spectrum Aggregation with Hardware Limitation in Cognitive Radio Networks”, IEEE VTC 2010-Spring, May 2010 